\title{
Ivermectin Treatment of Demodicosis in 56 Hamsters
}

\author{
Kenji TANI $^{1) *}$, Takeshi IWANAGA ${ }^{2)}$, Kai SONODA ${ }^{2)}$, Sanae HAYASHIYA ${ }^{1) *}$, Makio HAYASHIYA ${ }^{1)}$ and \\ Yasuho TAURA ${ }^{3) * *}$ \\ ${ }^{1)}$ Hayashiya Animal Hospital, 39-1205 Okurayama, Kohata, Uji 602-0002, ${ }^{2}$ Hayashiya Animal Hospital Momo, Yamanoshita, \\ Momoyama-cho, Fushimi-ku, Kyoto 612-8002 and ${ }^{3)}$ Department of Veterinary Surgery, Faculty of Agriculture, Yamaguchi University, \\ 1677-1 Yoshida, Yamaguchi 753-8515, Japan
}

(Received 2 March 2001/Accepted 30 July 2001)

\begin{abstract}
Fifty-six hamsters with demodicosis were treated with daily oral administration of ivermectin $(0.3 \mathrm{mg} / \mathrm{kg})$. Thirty-three cases (58.9\%) were cured and 6 cases $(10.7 \%)$ had improved clinically but needed to continue treatment. Of 5 cases (8.9\%) who relapsed within 3 months and were retreated with ivermectin, 4 were cured and 1 needed further treatment. Five cases $(8.9 \%)$ had improved clinically but died within 3 months. Seven cases (12.5\%) had not improved and died within 3 months. Overall $49(87.5 \%)$ hamsters had improved clinically. No significant differences in prognosis of demodicosis were detected according to sex, breed, age and clinical features, but the prognosis of demodicosis in hamsters with concurrent disease was poor.
\end{abstract}

KEY WORDS: demodicosis, hamster, ivermectin.

J. Vet. Med. Sci. 63(11): 1245-1247, 2001

In hamsters, demodex is thought to be a common parasite $[7,8,11,13,15]$ and to cause demodicosis associated with immunosuppressive disorders $[3,13,15,16]$, but this latter conclusion is drawn from single case reports or studies of hamsters kept under experimental conditions, and clinical confirmation of underlying disease is often deficient or lacking. In addition, there is little information regarding the response to treatment of demodicosis in hamsters [4]. Demodicosis in dogs is usually treated with amitraz [1], but most topical drugs may be unsuitable for the treatment of demodicosis as they fail to reach the deep-seated immature stages of the mites [8]. Ivermectin treatment has been evaluated in dogs with generalized demodicosis $[6,9,10,12,14]$ and the efficacy of this drug has been reported [9, 14]. Topical treatment with amitraz seems to be effective in hamsters with demodicosis $[1,13,15]$, although the efficacy of amitraz varies from one animal to another and the duration of treatment is long for their life-span. The purpose of the present study was to evaluate the efficacy of ivermectin treatment in hamsters with demodicosis in Japan.

The veterinary records of all hamsters with demodicosis diagnosed between January 1997 and December 1999 in Kyoto, Japan were reviewed. Demodicosis was diagnosed in hamsters with alopecia associated with a large number of demodex mites visible microscopically in skin scrapings. Careful physical examination of all hamsters and ultrasonography or X-ray of some hamsters was performed, but extensive work-ups such as a CBC, serum biochemical analyses, or serum hormone levels were not performed. Fiftysix hamsters were treated with daily oral administration of

\footnotetext{
* PRESENT ADDRESS: The United School of Veterinary Sciences, Yamaguchi University, 1677-1 Yoshida, Yamaguchi 753-8515, Japan.

**Correspondence to: Taura, Y. Department of Veterinary Surgery, Faculty of Agriculture, Yamaguchi University, 1677-1 Yoshida, Yamaguchi 753-8515, Japan.
}

ivermectin $(0.3 \mathrm{mg} / \mathrm{kg})$. These hamsters were considered to be cured when all skin lesions caused by the demodex mites had resolved and the results of microscopic examination of skin scrapings remained negative for 3 months. The distribution of demodicosis in male and female hamsters was evaluated with the z-test. The influence of sex, breed, age, clinical features at initial presentation and concurrent disease on prognosis was evaluated with the Kruskal-Wallis test and/or Mann-Whitney U test. Differences at $P<0.05$ were considered to be statistically significant.

From the veterinary records (males: 384; females: 368), 56 hamsters were diagnosed with demodicosis and fit the criteria for inclusion in this study. The hamsters ranged from 4 to 36 months of age (mean age 14.4 months; $n=55$ ), with one hamster of unknown age. There were 32 djungarian hamsters (Phodopus sungorus), 17 roborovski hamsters (Phodopus roborovskii) and 7 golden hamsters (Mesocricetus auratus). Forty-two of the hamsters were male and 14 were female. Demodicosis occurred more frequently in males than in females $(P<0.01)$. Thirty-two hamsters had mild-to-moderate pruritus, 21 had no pruritus, and the status of the remaining three at first referral was unknown. The location of the skin disease was abdominal $(n=22)$, dorsal $(n=10)$, lumbar $(n=9)$, neck $(n=8)$, axilla $(n=6)$, limb $(n=3)$ and generalized $(n=2)$. The most common skin site affected in this study was the abdomen, although the dorsum is thought to be the usual site affected $[2,8]$. Concurrent disease was diagnosed in 25 hamsters: pyoderma $(n=13)$, tumor $(n=5)$, Malassezia dermatitis $(n=5)$, malocclusion $(n=2)$, abscesses $(n=3)$, dermatophytosis $(n=2)$, congestive heart failure $(n=1)$, hamartoma $(n=1)$ and cataract $(n=1)$. The tumors in the 5 hamsters were diagnosed histopathologically as: fibrosarcoma $(n=2)$, lipoma $(n=1)$, sebaceous gland adenoma $(n=1)$ and sarcoma of unknown origin $(n=1)$.

Fifty-six hamsters were treated with ivermectin and were followed-up. Of these 56 hamsters, 33 (58.9\%) were cured 
Table 1. Results of oral administration of ivermectin to 56 hamsters with demodicosis

\begin{tabular}{|c|c|c|c|c|}
\hline \multirow[b]{2}{*}{ Classification } & \multicolumn{2}{|c|}{ Cleared* } & \multicolumn{2}{|c|}{ Not clear** } \\
\hline & Cured $\dagger$ & Relapsed $\ddagger$ & Need to treat $\mathbb{I}$ & Dead\# \\
\hline \multicolumn{5}{|l|}{ Sex } \\
\hline Female & 7 & 2 & 2 & 3 \\
\hline Male & 26 & 3 & 4 & 9 \\
\hline \multicolumn{5}{|l|}{ Breed (common name) } \\
\hline Djungarian hamster & 21 & 2 & 3 & 6 \\
\hline Roborovski hamster & 9 & 2 & 2 & 4 \\
\hline Golden hamster & 3 & 1 & 1 & 2 \\
\hline \multicolumn{5}{|l|}{ Age (months) } \\
\hline$<6$ & 2 & 0 & 0 & 0 \\
\hline $6 \leq,<12$ & 11 & 3 & 0 & 4 \\
\hline $12 \leq,<18$ & 12 & 0 & 4 & 2 \\
\hline $18 \leq,<24$ & 4 & 1 & 0 & 2 \\
\hline $24 \leq$ & 4 & 0 & 2 & 4 \\
\hline Unknown & 0 & 1 & 0 & 0 \\
\hline \multicolumn{5}{|l|}{ Pruritus } \\
\hline- & 13 & 0 & 2 & 6 \\
\hline+ & 19 & 4 & 3 & 6 \\
\hline Unknown & 1 & 1 & 1 & 0 \\
\hline \multicolumn{5}{|l|}{ Concurrent disease } \\
\hline$-\S$ & 23 & 3 & 2 & 3 \\
\hline+ & 10 & 2 & 4 & 9 \\
\hline Total & 33 & 5 & 6 & 12 \\
\hline
\end{tabular}

$*$ No mite seen microscopically in skin scraping. $\uparrow$ No clinical signs of demodicosis 3 months after administration of ivermectin were discontinued. $\ddagger$ Clinical signs of demodicosis returned after administration of ivermectin was discontinued. **No reduction in number of mites seen microscopically in skin scraping. If Clinical signs improved but need to treat. \# Dead for some reason within 3 months. $-=$ Absent. $+=$ Present. $§$ Significantly difference $(\mathrm{P}<0.01)$ for absent and present concurrent disease.

and $6(10.7 \%)$ were improved clinically but needed to continue treatment. Five $(8.9 \%)$ relapsed within 3 months and were retreated with ivermectin. Four of these were cured and one continued treatment. Five $(8.9 \%)$ improved clinically but died within 3 months. Seven $(12.5 \%)$ did not improve and died within 3 months. Tremor over the whole body was observed in one hamster, but this resolved shortly after treatment was stopped. This hamster was cured after restart of ivermectin treatment at a slightly lower dose $(0.2$ $\mathrm{mg} / \mathrm{kg})$. Overall, $49(87.5 \%)$ hamsters improved clinically. The duration of treatment for the 33 cured hamsters ranged from 7 to 49 days (mean 24 days). As shown in Table 1, no significant difference in prognosis of demodicosis was detected according to sex, breed, age or clinical features, but there was a significant difference in prognosis between hamsters with and without clinically concurrent disease $(P<0.01)$. Of the 12 hamsters which died within the 3 month study period, nine had concurrent disease (pyoderma, $n=3$; tumor, $n=3$; abscesses, $n=2$; Malassezia dermatitis, $n=1$; malocclusion, $\mathrm{n}=1$; hamartoma, $\mathrm{n}=1$ ). These hamsters ranged from 6 to 36 months of age (mean age 17.4 months).

Malnutrition, concurrent disease, presence of tumors and age have all been cited as predisposing factors for clinical manifestation of demodicosis in hamsters $[3,13,15,16]$. In this study no concurrent disease or underlying conditions were identified in 25 hamsters. Characteristic malnutrition was not detected and the most common concurrent diseases were skin diseases such as pyoderma, skin tumors, dermatophyte infection and cutaneous abscess. Only 5 cases of tumor were observed. Considering the age of onset, and compared with the number of hamsters with tumor in our veterinary hospital, this number was smaller than expected. Further analysis for abnormalities of the endocrine system, such as adrenal gland tumors or hyperplasia should be performed, although the size of the animals may preclude extensive pathological examination or endocrine testing [1].

It has been suggested that oral administration of ivermectin could reduce the side-effects associated with this drug [5], and ingestion of high dose $(0.6 \mathrm{mg} / \mathrm{kg})$ ivermectin has been used in some studies [9, 10, 14]. In this study, ivermectin was used at a dose of $0.3 \mathrm{mg} / \mathrm{kg}$ body weight to reduce side effects. Because hamsters have a larger surface area per unit body weight than dogs [16], higher doses of ivermectin may be more effective in hamsters with demodicosis, but it is emphasized that 12 of our hamsters died within the 3 month study period. This could reflect the toxicity of ivermectin in hamsters with underlying disorders. Further analysis of the optimum protocol for ivermectin treatment, including dose and duration, is needed, although there are some difficulties in evaluating the efficacy of such 
drugs because of the size and life-span of the hamster.

ACKNOWLEDGMENT. The authors would like to thank Dr. Chusnul for his critical suggestions during the preparation of the manuscript.

\section{REFERENCES}

1. Burke, T. J. 1992. pp. 1170-1175. In: Current Veterinary Therapy XI (Kirk, R. W. and Bonagura, J. D. eds.), W. B. Saunders, Philadelphia.

2. Collins, B. R. 1987. pp. 235-294. In: Dermatology (Nesbitt, G. H., ed), Churchill Livingstone.

3. Estes, P. C. and Richer, C. B. 1971. Lab. Anim. Sci. 21: 825828.

4. Hasegawa, T. 1995. J. Vet. Med. Sci. 57: 337-338.

5. Hsu, W. H., Wellborn, S. G. and Schaffer, C. B. 1989. Compendium. Small Anim. 11: 584-588.

6. Medleau, L., Ristic, Z. and Mcelveen, D. R. 1996. Vet. Dermatol. 7: 209-212.

7. Nutting, W. B. and Rauch, H. 1961. J. Parasitol. 47: 319-322.
8. Nutting, W. B. and Rauch, H. 1963. J. Parasitol. 49: 323-329.

9. Paradis, M. 1999. Vet. Clin. North. Am. Small Anim. Pract. 29: 1425-1436.

10. Ristic, Z., Medleau, L., Paradis, M. and White-Weithers, N. E. 1995. J. Am. Vet. Med. Assoc. 207: 1308-1310.

11. Sarashina, T. and Sato, K. 1986. Jpn. J. Vet. Sci. 48: 619-622.

12. Scott, D. W., Miller, W. H., Jr. and Griffin, C. E. 2001. pp. 423-516. In: Muller and Kirk's Small Animal Dermatology, 6th ed. (Scott, D. W., Miller, W. H., Jr. and Griffin, C. E. eds.), W. B. Saunders, Philadelphia.

13. Scott, D. W., Miller, W. H., Jr. and Griffin, C. E. 2001. pp. 1415-1458. In: Muller and Kirk's Small Animal Dermatology, 6th ed. (Scott, D. W., Miller, W. H., Jr. and Griffin, C. E. eds.), W. B. Saunders, Philadelphia.

14. Tani, K., Hayashiya, S. and Taura Y. 2000. J. Jpn. Vet. Med. Assoc. 53: 71-74 (in Japanease with English abstract)

15. Tim, K. I. 1988. Vet. Clin. North. Am. Small Anim. Pract. 18: 1077-1091.

16. Wagner, J. E. and Farrar, P. L. 1987. Vet. Clin. North. Am. Small Anim. Pract. 17: 1061-1087. 